\title{
Early metabolic response using FDG PET/CT and molecular phenotypes of breast cancer treated with neoadjuvant chemotherapy
}

Bhumsuk Keam ${ }^{1,2}$, Seock-Ah Im ${ }^{1,2^{*}}$, Youngil Koh' ${ }^{1}$, Sae-Won Han ${ }^{1,2}$, Do-Youn Oh ${ }^{1,2}$, Nariya Cho ${ }^{4}$, Jee Hyun Kim ${ }^{1,2}$, Wonshik Han ${ }^{5}$, Keon Wook Kang ${ }^{2,3}$, Woo Kyung Moon ${ }^{4}$, Tae-You Kim ${ }^{1,2}$, In Ae Park ${ }^{6}$, Dong-Young Noh ${ }^{5}$, June-Key Chung ${ }^{2,3}$ and Yung-Jue Bang ${ }^{1,2}$

\begin{abstract}
Background: This study was aimed 1) to investigate the predictive value of FDG PET/CT (fluorine-18 fluorodeoxyglucose positron emission tomography/computed tomography) for histopathologic response and 2) to explore the results of FDG PET/CT by molecular phenotypes of breast cancer patients who received neoadjuvant chemotherapy.

Methods: Seventy-eight stage II or III breast cancer patients who received neoadjuvant docetaxel/doxorubicin chemotherapy were enrolled in this study. FDG PET/CTs were acquired before chemotherapy and after the first cycle of chemotherapy for evaluating early metabolic response.

Results: The mean pre- and post-chemotherapy standard uptake value (SUV) were 7.5 and 3.9, respectively. The early metabolic response provided by FDG PET/CT after one cycle of neoadjuvant chemotherapy was correlated with the histopathologic response after completion of neoadjuvant chemotherapy $(P=0.002)$. Sensitivity and negative predictive value were $85.7 \%$ and $95.1 \%$, respectively. The estrogen receptor negative phenotype had a higher pre-chemotherapy SUV (8.6 vs. $6.4, P=0.047$ ) and percent change in SUV (48\% vs. $30 \%, P=0.038$ ). In triple negative breast cancer (TNBC), the pre-chemotherapy SUV was higher than in non-TNBC (9.8 vs. 6.4, $P=0.008$ ).

Conclusions: The early metabolic response using FDG PET/CT could have a predictive value for the assessment of histopathologic non-response of stage II/III breast cancer treated with neoadjuvant chemotherapy. Our findings suggest that the initial SUV and the decline in SUV differed based on the molecular phenotype.
\end{abstract}

Trial Registration: ClinicalTrials.gov: NCT01396655

Keywords: FDG PET, breast cancer, neoadjuvant chemotherapy, molecular phenotype

\section{Background}

Breast cancer is second most common cancer in Korean women [1]. Neoadjuvant chemotherapy, also called preoperative chemotherapy, has emerged as the preferred initial component of therapy for patients diagnosed with locally advanced breast cancer. In the treatment of locally advanced breast cancer, multimodality approach, including neoadjuvant chemotherapy, surgery, and radiotherapy, is needed [2-4].

\footnotetext{
* Correspondence: moisa@snu.ac.kr

'Department of Internal Medicine, Seoul National University College of Medicine, Seoul, Korea

Full list of author information is available at the end of the article
}

Over the last decade, the clinical value of fluorine-18 fluorodeoxyglucose positron emission tomography (FDG PET) in breast cancer has been widely studied [5-8]. However, a few studies have been conducted in stage II or III breast cancer patients receiving neoadjuvant chemotherapy, and the role of FDG PET remains inconclusive because of the small number of patients and heterogeneous regimens [9-13].

Furthermore, breast cancer is a heterogeneous disease with a demonstrated difference in prognosis based on molecular phenotypes. Many researchers have attempted to perform risk stratification and individualized treatment according to molecular phenotypes. We conducted

\section{Biomed Central}


a prospective phase II study of neoadjuvant docetaxel/ doxorubicin chemotherapy in stage II or III breast cancer and recently reported the prognostic and predictive role of the molecular markers $[14,15]$.

However, little is known how we consider the molecular phenotypes of breast cancer in order to interpret FDG PET findings [16]. This lead us to further investigate FDG PET in stage II or III breast cancer patients who received neoadjuvant chemotherapy, and FDG PET/CT was performed as a part of large clinical phase II study. The objectives of this study were 1) to investigate the predictive value of FDG PET/CT for histopathologic responses and 2) to explore the results of FDG PET/CT by molecular phenotypes of breast cancer.

\section{Methods}

\section{Patients and treatment}

Between July 2006 and September 2008, a total of 78 stage II or III breast cancer patients who received neoadjuvant docetaxel/doxorubicin chemotherapy were enrolled in this prospective study. The primary endpoint of this trial was evaluating pathologic complete response ( $\mathrm{pCR}$ ) rate, because $\mathrm{pCR}$ is repeatedly confirmed most relievable prognostic factor for neoadjuvant chemotherapy $[17,18]$, and the best predictor of improved outcome and prolonged survival [3]. Secondary endpoints were evaluating survival, toxicity, predictive factors, and early metabolic response. The eligibility criteria were described in our prior reports $[14,15]$. In brief, the eligibility criteria were as follows: 1 ) pathologically-confirmed breast cancer by core needle biopsy, 2) initial clinical stage II or III, 3) objective measurable lesion, 4) ECOG performance 0 2, 5) previously untreated, and 6) adequate bone marrow, hepatic, cardiac, and renal functions. The patients received three cycles of neoadjuvant docetaxel/doxorubicin chemotherapy. The chemotherapeutic regimen consisted of docetaxel $(75 \mathrm{mg} /$ $\mathrm{m}^{2}$ ) and doxorubicin $\left(50 \mathrm{mg} / \mathrm{m}^{2}\right)$ by intravenous infusion every 3 weeks. After three cycles of neoadjuvant chemotherapy, the patients were re-evaluated for response and underwent curative surgery. Radiologic response was evaluated using breast magnetic resonance imaging (MRI) for the primary breast tumor and chest computed tomography (CT) for axillary, supraclavicular, internal mammary lymph nodes with RECIST criteria [19]. Both breast MRI and chest CT were performed in all the 78 patients. Subsequently, the patients received three more cycles of docetaxel and doxorubicin as an adjuvant chemotherapy, followed by hormonal or radiation therapy, if indicated [20]. Figure 1 shows the scheme for this study protocol. This regimen is known to be effective as neoadjuvant chemotherapy for stage II or III breast cancer [14,15,21]. We performed retrospective descriptive analysis for PET/CTs within this prospective study. This study protocol was conducted under the approval of the Institutional Review
Board of Seoul National University Hospital (IRB approval number: 0510-506-159).

\section{Pathologic Assessment}

The molecular markers that we have examined included estrogen receptor (ER), progesterone receptor (PR), human epidermal growth factor receptor 2 (HER2), and Ki-67. We performed immunohistochemistry (IHC) using tissues obtained before treatment and evaluated the association with clinical outcomes. IHC was performed as previously described $[15,22]$. The cut-off for ER and PR positivity was $\geq 10 \%$ positive tumor cells with nuclear staining. HER2 positivity was defined as either HER2 gene amplification by fluorescent in situ hybridization or scored as $3+$ by IHC [23]. The molecular phenotypes of breast cancer were classified into luminal A, luminal B, HER2 and triple negative breast cancer (TNBC) [24]. TNBC was defined as ER(-), PR(-), and HER2(-). Luminal A phenotype was defined as $\mathrm{ER}(+)$ or $\mathrm{PR}(+)$ and HER2(-) tumor, and luminal $\mathrm{B}$ phenotype was defined as $\mathrm{ER}(+)$ or $\mathrm{PR}(+)$ and HER2(+). ER(-) and PR (-) and HER2+ tumor was classified as HER2 phenotype. Low expression of Ki-67 was defined as $\leq 5 \%$. Histopathologic response was classified as proposed by Honkoop et al. [25] Specimens with no residual invasive carcinoma were classified as pCR. Residual ductal carcinoma in situ was included in the pCR category [26]. Specimens with only few scattered foci of microscopic residual invasive tumor were classified as minimal residual disease (MRD). Gross residual disease (GRD) comprised tumors showing macroscopic residual tumor or extensive residual tumor infiltration on microscopic examination. $\mathrm{pCR}$ and MRD were defined as histopathologic response and GRD as no histopathologic response.

\section{FDG PET/CT studies}

Whole-body fluorine-18 FDG PET/CT scans were acquired before and after neoadjuvant chemotherapy for early metabolic response prediction. On the $15^{\text {th }}$ day of the first cycle of neoadjuvant chemotherapy, we obtained follow-up whole-body fluorine-18 FDG PET/CT scans. The CT scan protocol was as follows: tube voltage, 120 kV; current intensity, 50 mAs; scan time, 43.2 seconds; effective radiation dose, $4.8 \mathrm{mSv}$. FDG PET/CT was done using the same scanner (Gemini PET/CT system; Philips, Milpitas, CA, USA). Patients fasted for at least 6 hours before fluorine-18 FDG was injected intravenously. Patients were administered a weight-adjusted dosed of fluorine-18 FDG $(5.18 \mathrm{MBq} / \mathrm{kg})$, and images were acquired approximately 60 minutes (range: 50-75 minutes) after an intravenous injection of fluorine-18 FDG. Whole body emission scans were obtained for 2 minutes per bed position. PET/CT scanners automatically calculated the decay-corrected injected activity. We flushed 


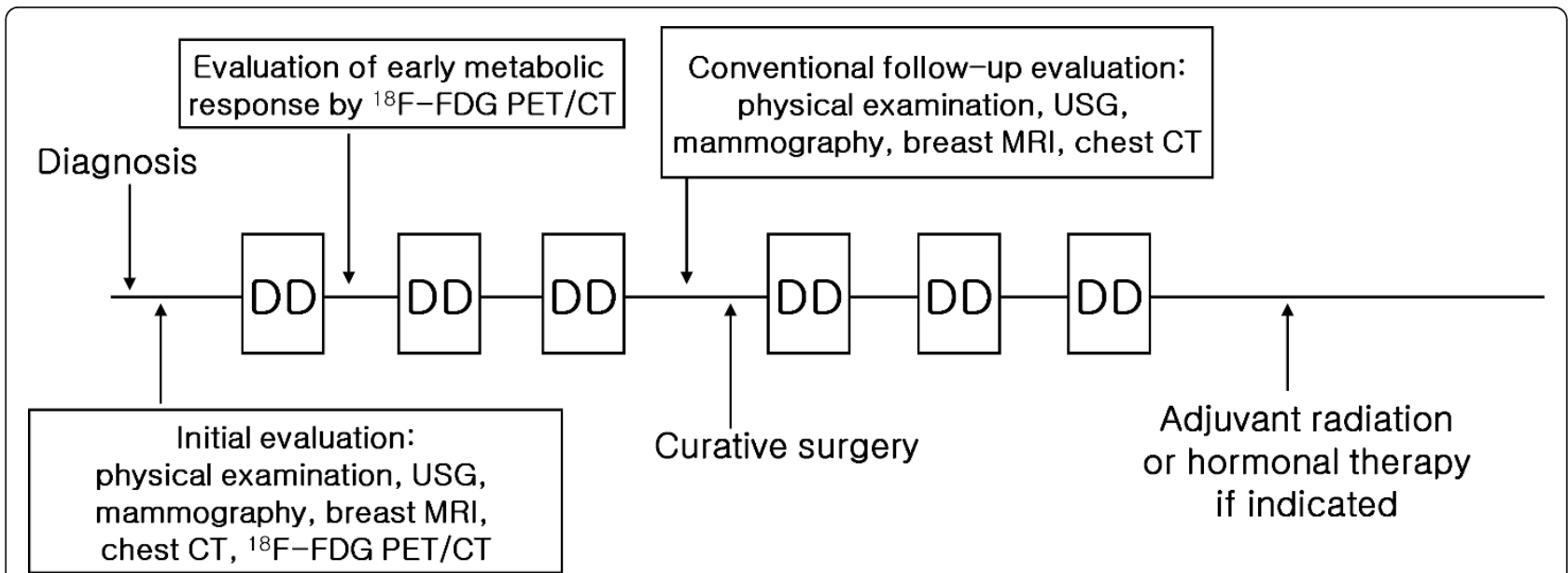

Figure 1 Schematic flow of the neoadjuvant chemotherapy and response assessed by FDG PET/CT, breast MRI and chest CT. Abbreviations: DD, docetaxel+doxorubicin; FDG PET, fluorine-18 fluorodeoxyglucose positron emission tomography; CT, computed tomography; MRI, magnetic resonance imaging; USG, ultrasonography.

the syringe and venous catheter three times, and residual activities in the syringe were less than $20 \mu \mathrm{Ci}$ and negligible. All patients were studied in the supine position with both arms raised above the head to pull their breast away.

Attenuation correction was based on the CT data, and PET data reconstruction was done by a 3-dimensional row action maximum likelihood algorithm. For quantitative assessment of tumor FDG uptake, region of interest were manually drawn on the slice with the highest radioactivity concentration of the primary breast tumor and in the adjacent slices.

The standard uptake values (SUVs) were calculated from the amount of FDG injected, body weight, and soft tissue uptake in the attenuation-corrected regional images as follows: SUV = (activity/unit volume)/(injected dose/body weight). Maximal SUV was defined as the SUV value on one pixel with the highest counts within the region of interest. The lesion chosen for analysis was the primary breast lesion with the highest SUV. The pre-treatment baseline maximal SUV of the tumor (pre-SUV) and the SUV of the tumor after the first cycle of chemotherapy (post-SUV) were compared with clinico-pathologic parameters. Calculation of the uptake index was as follows: $\Delta \mathrm{SUV} \%=100 \times($ pre-SUV - post-SUV $) /$ pre-SUV.

\section{Statistical analysis}

This is a subproject of a phase II trial. The primary endpoint of the main project was $\mathrm{pCR}$, and we designed this trial to test null hypothesis that the $\mathrm{pCR}$ rate was at most $10 \%$ against the alternative hypothesis that it was at least $20 \%$. A sample size of 78 patients provided $80 \%$ power to test this hypothesis and $\alpha 0.05$, using Simon's two stage minimax design [27]. The statistical consideration was performed according to the main project. The subproject has investigated the early metabolic change as primary endpoint. This was an exploratory analysis only, without an own sample size calculation for subproject. The primary objective of this subproject was to find a correlation between early metabolic response and pathological response. The association between histopathologic and metabolic response were assessed by a chi-square test. The cut-off of the SUV change for metabolic response was not prospectively defined. Receiver-operating characteristics (ROC) analysis was performed to determine a threshold for the prediction of metabolic response. The MannWhitney U test or Kruskal-Wallis test were used to compare SUVs according to different molecular phenotypes. All statistical tests were two-sided, with the level of significance established at $P<0.05$. SPSS software (SPSS, Inc., Chicago, IL, USA) was used for all statistical analyses.

\section{Results}

\section{Patients and results of treatment}

The pretreatment characteristics are given in Table 1. Twelve patients $(15.7 \%)$ were initially staged clinical stage II and the others (84.3\%) were initial staged clinical stage III. The median follow-up duration was 18.7 months (range, 4.9-30.9 months). The tumor size was a median of $4.5 \mathrm{~cm}$ in the greatest dimension (range, $2.0-11.0 \mathrm{~cm}$ ). At the end of follow-up, eight patients had developed recurrent disease, and one patient had died. The overall histopathologic response rate was $17.9 \%$. Table 2 shows the results to neoadjuvant chemotherapy.

\section{Changes in FDG PET/CT during neoadjuvant chemotherapy}

The mean maximal SUVs \pm standard deviation (SD) were as follows: pre-SUV $=7.5 \pm 4.3$ (range, 1.4 22.1), 
Table 1 Baseline characteristics of 78 patients

\begin{tabular}{|c|c|}
\hline Characteristics & No. of Pt (\%) \\
\hline Median age (range) & 45 (range 29-69) \\
\hline Age $<50$ & $56(71.8)$ \\
\hline Age $\geq 50$ & $22(28.2)$ \\
\hline \multicolumn{2}{|l|}{ Performance status } \\
\hline ECOG 0 & $23(29.5)$ \\
\hline ECOG 1 & $55(70.5)$ \\
\hline \multicolumn{2}{|l|}{ Pathologic characteristics } \\
\hline Invasive ductal carcinoma & $74(94.9)$ \\
\hline Others & $4(5.1)$ \\
\hline \multicolumn{2}{|l|}{ Initial clinical stage } \\
\hline$\| \mathrm{A}$ & $1(1.3)$ \\
\hline$\| B$ & $11(14.1)$ \\
\hline$\| \mathrm{A}$ & $47(60.3)$ \\
\hline$\| \mathrm{IIB}$ & $13(16.7)$ \\
\hline IIIC & $6(7.7)$ \\
\hline \multicolumn{2}{|l|}{ Inflammatory breast cancer } \\
\hline Yes & $3(3.8)$ \\
\hline No & $75(96.2)$ \\
\hline \multicolumn{2}{|l|}{ Type of surgery } \\
\hline Breast conserving & $44(56.4)$ \\
\hline Mastectomy & $34(43.6)$ \\
\hline \multicolumn{2}{|l|}{ Adjuvant hormonal therapy } \\
\hline Yes & $52(66.7)$ \\
\hline No & $26(33.3)$ \\
\hline \multicolumn{2}{|l|}{ Adjuvant radiation therapy } \\
\hline Yes & $66(84.6)$ \\
\hline No & $12(15.4)$ \\
\hline
\end{tabular}

ECOG, Eastern Cooperative Oncology Group.

post-SUV $=3.9 \pm 2.7$ (range, $0 \sim 12$ ), mean $\Delta$ SUV\% $=$ $40 \%$ (range, $-260 \% \sim 100 \%$; SD $=46$ ). The treatment response was assessed by breast MRI and chest $\mathrm{CT}$ for radiologic response and by FDG PET/CT for early metabolic response. $\triangle$ SUV\% and decrease \% of tumor diameter measured by RECIST criteria were well correlated (Pearson correlation coefficient $=0.267, P=0.019$ ) .

ROC analysis were performed to determine optimal cut-off values of $\triangle$ SUV\% to differentiate metabolic responder and non-responder, based on histopathologic response (Figure 2). The cut-off to achieve the highest positive predictive value was $\Delta$ SUV\% of $50 \%$ (sensitivity of $85.7 \%$, specificity of $60.9 \%$ ). Among the all 78 patients,

Table 2 Results to docetaxel plus doxorubicin neoadjuvant chemotherapy

\begin{tabular}{lc}
\hline Response & No. of Pts (\%) \\
\hline Histopathologic response & \\
Pathologic complete response & $4(5.1)$ \\
Minimal residual disease & $10(12.8)$ \\
Gross residual disease & $64(82.1)$ \\
\hline
\end{tabular}

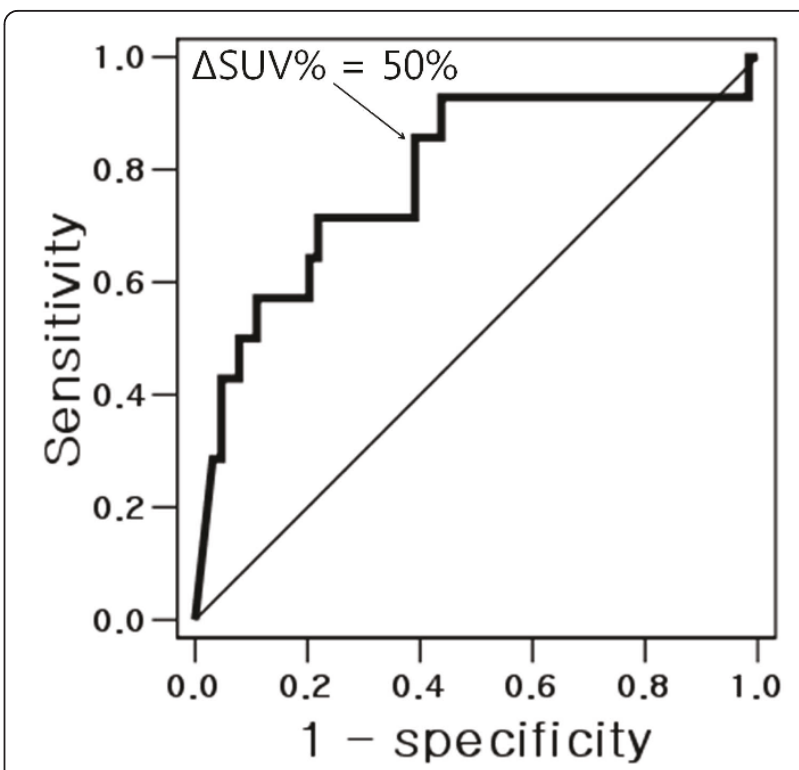

Figure 2 Receiver-operating characteristics (ROC) analysis, determine optimal cut-off values of $\Delta \mathrm{SUV} \%$; Area under the curve ROC was 0.79 (95\% confidence interval 0.64-0.93).

37 patients $(47.4 \%)$ achieved metabolic response, while 41 patients $(52.6 \%)$ did not.

$\triangle$ SUV\% was significantly greater in patients with histopathologic response than patients without histopathologic response (63\% vs. $34 \%, P=0.001$, Table 3$)$. PreSUVs were not different between histopathologic responder and non-responder, however post-SUVs were significantly differed, and this led to a difference of $\Delta$ SUV\%. Histopathologic response was higher in early metabolic responders determined by FDG PET/CT after one cycle of neoadjuvant chemotherapy $(P=0.002)$ (Table 4). Among 14 histopathologic responder, 12 (85.7\%) showed metabolic response. Importantly, 39 patients among the 41 metabolic non-responders (95.1\%) were eventually revealed as histopathologic nonresponder (Table 4 ).

\section{Changes in FDG PET/CT and histopathologic response according to molecular phenotype}

The FDG PET/CT results were different according to the molecular phenotype of the breast cancer. The ER

Table 3 Correlation between response and SUV values.

\begin{tabular}{lccc}
\hline Response & Pre-SUV & Post-SUV & $\Delta$ SUV\% \\
\hline Histopathologic response & & & \\
$\quad$ Responders & $7.3 \pm 4.2$ & $2.2 \pm 1.9$ & $63 \pm 48$ \\
Non-responders & $7.6 \pm 4.4$ & $4.3 \pm 2.7$ & $34 \pm 47$ \\
& $P=0.795$ & $P=0.004$ & $P=0.001$ \\
\hline
\end{tabular}

SUV, standard uptake value

$P$-value based on the Mann-Whitney $U$ test, presuming to be non-parametric statistics 
Table 4 Correlation between metabolic, and histopathologic response.

\begin{tabular}{lcc}
\hline Response & Histopathologic responders * & $\begin{array}{c}\text { Histopathologic } \\
\text { non-responders }\end{array}$ \\
\hline $\begin{array}{l}\text { Metabolic responders } \\
\text { Responders }\end{array}$ & $12(32.4 \%)$ & $25(67.6 \%)$ \\
Non-responders & $2(4.9 \%)$ & $39(95.1 \%)$ \\
\hline & For histopathologic responders & For histopathologic nonresponder \\
\hline Sensitivity & $85.7 \%(12 / 14)$ & $60.9 \%(39 / 64)$ \\
Specificity & $60.9 \%(39 / 64)$ & $85.7 \%(12 / 14)$ \\
Positive predictive value & $32.4 \%(12 / 37)$ & $95.1 \%(39 / 41)$ \\
Negative predictive value & $95.1 \%(39 / 41)$ & $32.4 \%(12 / 37)$ \\
\hline
\end{tabular}

* Pathologic complete response or minimal residual disease were regarded as histopathologic responders, Gross residual disease were regarded as histopathologic non-responders.

${ }^{+} P$-value based on the Fisher's exact test

negative phenotype showed a higher pre-SUV (8.6 vs. $6.4, P=0.047)$ and $\triangle \mathrm{SUV} \%(48 \%$ vs. $30 \%, P=0.038)$ than ER positive phenotype. In patients with high Ki-67 expression, a similar phenomenon was observed in that the pre-SUV and $\triangle$ SUV\% were higher than in the low Ki-67 expression group (8.5 vs. 6.2, $P=0.018 ; 49 \%$ vs. $27 \%, P=0.052$, respectively). In the TNBC group, the pre-chemotherapy SUV was higher than in the nonTNBC group (9.8 vs. 6.4, $P=0.008$ ); however, the $\Delta$ SUV\% was not different $(P=0.799)$. Table 5 and Figure 3 show the serial FDG PET/CT results according to molecular phenotypes. Histopathologic response rate was higher in ER negative patients than ER positive patients $(27.5 \%$ vs. $7.9 \%, P=0.037)$ and early metabolic response rate tend to higher in ER negative patients (57.5\% vs. $36.8 \%, P=0.068$ ). In this context, histopathologic response rate was significantly different according to molecular phenotype $(P=0.018)$ and metabolic response tend to higher in HER2+ and TNBC subtypes than luminal type (66.7\% vs. $50.0 \%$ vs. $40.0 \%, P=0.255)$. Sensitivity and specificity of metabolic response for histopathologic response were higher in ER positive phenotype $(100.0 \%$ and $68.6 \%$, respectively) than those of ER negative phenotype ( $81.8 \%$ and $51.7 \%$, respectively).

\section{Discussion}

In the present study, we found that the early metabolic response using serial FDG PET/CT had a high predictive value for the histopathologic non-response of stage II/III breast cancer treated with neoadjuvant chemotherapy. Our study has demonstrated that the early metabolic response obtained on the $15^{\text {th }}$ day of first cycle of neoadjuvant chemotherapy may be useful in determining histopathologic non-responders with high negative predictive value of $95.1 \%$, however, its value to identify histopathologic responders was low with positive predictive value of 32.4\%. Different molecular phenotypes based on IHC reflect different metabolic properties and different histopathologic response rate.
Because current methods to monitoring tumor response (i.e., physical examination and conventional imaging modalities) have potential limitations [12], early metabolic responses by serial FDG PET/CT would be a useful complementary tool. Assessment of therapeutic response with serial FDG PET/CT scans was possible earlier than with any other method. Early evaluation of chemosensitivity in vivo using serial FDG PET/CT would help to identify non-responding patients, to avoid ineffective chemotherapy $[28,29]$ and to decide the most appropriate therapeutic strategy. With given sensitivity of $85.7 \%$ and negative predictive value of $95.1 \%$, metabolic non-responders identified by early serial FDG PET/CTs were likely to be histopathologic non-responders. In order to improve treatment outcome by risk stratification, the metabolic non-responder could be considered to have received another treatment strategy in earlier time course. It is necessary to design a future prospective randomized clinical trial comparing continuing standard cytotoxic chemotherapy alone versus incorporating new agents for metabolic non-responders to decide the clinical value of early determination of metabolic nonresponse.

Our study confirms previous reports [9,10,12,13,29-36] on the predictive value of changes in glucose metabolism after neoadjuvant chemotherapy in a prospective setting. Rousseau et al. [12] reported sensitivity of $61 \%$, specificity of $96 \%$, and negative predictive value of $68 \%$ when using FDG PET/CT after the first cycle of chemotherapy with a SUV cut-off of $60 \%$. When comparing PET/CTs obtained baseline and 2nd cycles of chemotherapy, sensitivity and specificity were reported $73 \%$ and $63 \%$ with a cut-off of $45 \%$ [35], 93\% and 75\% with a cut-off of 50\% [29], 77\% and $80 \%$ with a cut-off of $40 \%$ [31]. The previous studies using FDG PET in neoadjuvant settings have indicated that measurements of SUV or changes in SUV during neoadjuvant chemotherapy allow prediction of histopathologic response as well as survival [9,10,12,13,29-37]. We used $\triangle$ SUV\% because it was found to be the only 
Table 5 SUV and changes in SUV based on molecular phenotypes

\begin{tabular}{|c|c|c|c|c|c|}
\hline & No. of Pts & Pre-SUV * & Post-SUV * & $\Delta$ SUV\% $*$ & HPR (\%) \\
\hline \multicolumn{6}{|l|}{ ER } \\
\hline Positive & 38 & $6.4 \pm 3.3$ & $3.7 \pm 2.1$ & $30 \pm 57$ & $3(7.9)$ \\
\hline \multirow[t]{2}{*}{ Negative } & 40 & $8.6 \pm 4.9$ & $4.1 \pm 3.1$ & $48 \pm 37$ & $11(27.5)$ \\
\hline & & $P=0.047$ & $P=0.936$ & $P=0.038$ & $P=0.037^{\dagger}$ \\
\hline \multicolumn{6}{|l|}{ PR } \\
\hline Positive & 30 & $6.5 \pm 3.3$ & $3.5 \pm 2.4$ & $36 \pm 63$ & $3(10.0)$ \\
\hline \multirow[t]{2}{*}{ Negative } & 48 & $8.2 \pm 4.8$ & $4.2 \pm 2.8$ & $42 \pm 37$ & $11(22.9)$ \\
\hline & & $P=0.187$ & $P=0.347$ & $P=0.837$ & $P=0.226^{\dagger}$ \\
\hline \multicolumn{6}{|l|}{ HER2 } \\
\hline Positive & 17 & $6.8 \pm 3.2$ & $3.2 \pm 2.6$ & $51 \pm 36$ & $5(29.4 \%)$ \\
\hline \multirow[t]{2}{*}{ Negative } & 61 & $7.7 \pm 4.6$ & $4.1 \pm 2.7$ & $36 \pm 51$ & $9(14.8 \%)$ \\
\hline & & $P=0.818$ & $P=0.247$ & $P=0.189$ & $P=0.164^{\dagger}$ \\
\hline \multicolumn{6}{|l|}{ Triple negativity } \\
\hline TNBC & 26 & $9.8 \pm 5.3$ & $5.0 \pm 3.0$ & $42 \pm 35$ & $6(23.1)$ \\
\hline \multirow[t]{2}{*}{ Non-TNBC } & 52 & $6.4 \pm 3.2$ & $3.4 \pm 2.3$ & $38 \pm 54$ & $8(15.4)$ \\
\hline & & $P=0.008$ & $P=0.076$ & $P=0.799$ & $P=0.404^{\dagger}$ \\
\hline \multicolumn{6}{|l|}{ Molecular phenotype } \\
\hline Luminal $A / B$ & 40 & $6.3 \pm 3.3$ & $3.6 \pm 2.2$ & $33 \pm 57$ & $3(7.5)$ \\
\hline HER2 & 12 & $6.6 \pm 3.2$ & $2.8 \pm 2.6$ & $56 \pm 39$ & $5(41.7)$ \\
\hline \multirow[t]{2}{*}{ TNBC } & 26 & $9.8 \pm 5.3$ & $5.0 \pm 3.0$ & $42 \pm 35$ & $6(23.1)$ \\
\hline & & $P=0.016^{\ddagger}$ & $P=0.055^{\ddagger}$ & $P=0.150^{\ddagger}$ & $P=0.018^{\dagger}$ \\
\hline \multicolumn{6}{|l|}{ Ki-67 } \\
\hline Low expression $\$$ & 30 & $6.2 \pm 4.1$ & $3.6 \pm 2.6$ & $27 \pm 65$ & $4(13.3)$ \\
\hline \multirow[t]{2}{*}{ High expression } & 41 & $8.5 \pm 4.4$ & $4.0 \pm 2.8$ & $49 \pm 33$ & $10(24.4)$ \\
\hline & & $P=0.018$ & $P=0.541$ & $P=0.052$ & $P=0.367^{\dagger}$ \\
\hline
\end{tabular}

HPR, histopathologic responder; ER, estrogen receptor; PR, progesterone receptor; HER2, human epidermal growth factor receptor 2; TNBC, triple negative breast cancer.

* Data are presented as the mean \pm standard deviation.

${ }^{+} P$-values are based on Chi square or Fisher's exact test.

‡ $P$-values are based on Kruskal-Wallis test, otherwise $P$-values are based on Mann-Whitney $U$ test, presuming to be non-parametric statistics.

$\S$ Low expression of $\mathrm{Ki}-67$ was defined as $\leq 5 \%$, and 7 patients were not available for $\mathrm{Ki}-67$ results.

independent predictive factor of histopathologic response in previous report [30]. Histopathologic response was higher in metabolic responder compared with metabolic non-responder (32.4\% vs. 4.9\%) although substantial portion of metabolic responder did not eventually get histopathologic response. Lower histopathologic response rate in our study was attributed to relatively short course of neoadjuvant chemotherapy in relatively advanced large tumors (median tumor size $4.5 \mathrm{~cm}$ ). Importantly, 39 out of 41 metabolic non-responders did not get pathologic response which makes high negative predictive value (95.1\%), and $85.7 \%$ of metabolic responders eventually showed pathologic response.

Bos et al. [16] reported that ${ }^{18}$ FDG uptake in breast cancer was determined by the several biologic markers including glucose transporter-1, hexokinase I expression and mitotic activity index in breast cancer. Avril et al. [38] analyzed 56 operable breast cancer patients, and reported the positive correlation between baseline FDG uptake and tumor cell proliferation, which were similar with our results. In that report, hormone receptor negative breast cancer tended to have higher pre-SUV level than hormone receptor positive breast cancer, even though not reached statistical significance. However, little is currently known about the correlation between the SUV changes of FDG PET results and the meaning of molecular phenotype which is commonly used in the clinic to decide the treatment plan. In our study, breast cancer patients with high expression of Ki-67, which is regarded as highly proliferative and aggressive [39], showed a higher pre-SUV. ER negativity and high expression of Ki-67 was also associated with a high declining of the SUV. The pre-chemotherapy SUV of TNBC was higher than in non-TNBC, which was in line with other study [40].

Our study has some limitation. First, the median follow-up duration is relatively short with median follow-up duration of 18.7 months. As only few patients experienced relapses, we did not make an attempt to correlate the results of FDG PET/CT with survival. Second, the 

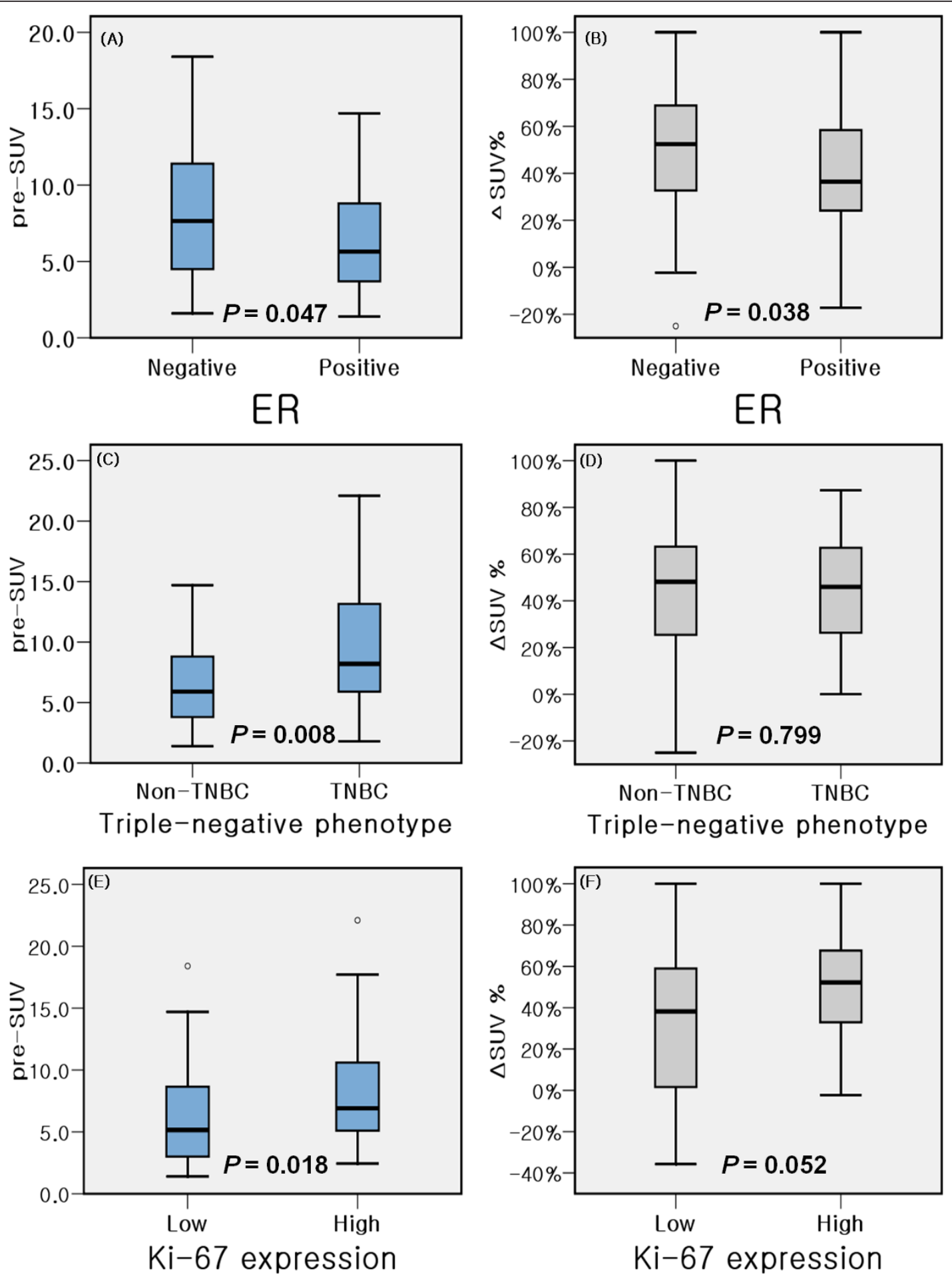

Figure 3 Box flows comparing serial FDG PET/CT results according to molecular phenotypes based on immunohistochemistry. ER negative phenotype showed higher pre-SUV (A) and $\triangle S U V \%$ (B) than ER positive phenotype. In patients with high Ki-67 expression, a similar phenomenon was observed in that pre-SUV (C) and $\triangle S U V \%$ (D) was higher than the low Ki-67 expression group. In triple negative breast cancer (TNBC), pre-SUV (E) was higher than non-TNBC; however, $\triangle$ SUV\% (F) was not different. 
cut-off value of $\Delta$ SUV\% should be validated in a larger study. Third, we did not obtain confirmatory statistical power for subgroup analysis.

However, our study was the first study exploring the metabolic uptake changes according to the clinically relevant molecular phenotype using serial FDG PET/CT before and after neoadjuvant chemotherapy. The patient population was highly homogenous in terms of stage and treated by same chemotherapy regimen and schedule. SUVs were obtained by the same PET/CT machine with the same protocol in a single institution. Metabolic lesions were well-matched because we used a combined PET/CT system.

\section{Conclusions}

Despite some limitations, this study suggests that early metabolic response assessment with FDG PET/CT after the first cycle of neoadjuvant chemotherapy accuratly predicted histopathologic non-response and could be useful to identify patients undergoing ineffective or needing more aggressive chemotherapy. We also observed that the initial SUV and declining of the SUV differed based on molecular phenotype. Further prospective studies confirming the retrospectively calculated cut-off of $50 \%$, and using longer duration of neoadjuvant chemotherapy with serial FDG PET/CT including early time point and just prior to definitive surgery are warranted.

\begin{abstract}
Abbreviations
FDG PET: fluorine-18 fluorodeoxyglucose positron emission tomography; $\mathrm{CT}$ : computed tomography; MRI: magnetic resonance imaging; ER: estrogen receptor; PR: progesterone receptor; HER2: human epidermal growth factor receptor 2; IHC: immunohistochemistry; TNBC: triple negative breast cancer; pCR: pathologic complete response; MRD: minimal residual disease; GRD: gross residual disease; SUV: standard uptake value; ROC: receiver-operating characteristics.
\end{abstract}

\section{Acknowledgements}

This work was supported by the Seoul National University Brain Fusion Program 2010 Research Grant to SA Im, and was also supported by Basic Science Research Program Through the National Research Foundation of Korea (NRF) funded by the Ministry of Education, Science and Technology (2010-0022299). We would like to thank our clinical fellows (Dr. Dae-Young Kim, Dr. Myung-Deok Seo, Dr. Jong-Mu Sun, and Dr. Hye Jung Chang) and clinical research nurses (Kyung-Hee Lee, Ji-Sook Kim, and Hyun Sook Kang) for their assistance in the clinical trial. We also thank BioMed Proofreading LLC for English editing. This study was presented as oral presentation at the 33rd European Society for Medical Oncology Congress in Stockholm, Sweden (1216 September 2008).

\section{Author details}

${ }^{1}$ Department of Internal Medicine, Seoul National University College of Medicine, Seoul, Korea. ${ }^{2}$ Cancer Research Institute, Seoul National University College of Medicine, Seoul, Korea. ${ }^{3}$ Department Nuclear Medicine, Seoul National University College of Medicine, Seoul, Korea. ${ }^{4}$ Department of Radiology, Seoul National University College of Medicine, Seoul, Korea. ${ }^{5}$ Department of Surgery, Seoul National University College of Medicine, Seoul, Korea. ${ }^{6}$ Department of Pathology, Seoul National University College of Medicine, Seoul, Korea.

\section{Authors' contributions}

Designing the concept of the study: SAI, BK. Provision of study patients and chemotherapy: SAI, SWH, DYO, JHK, TYK, YJB. Provision of study patients and surgery: WH, DYN. Pathologic examination and immunohistochemistry: IAP. Image analysis: NC, KWK, WKM, JKC. Data gathering, statistical analysis and interpretation: BK, YK. Manuscript writing: SAI, BK. All authors read and approved the final manuscript

\section{Competing interests}

The authors declare that they have no competing interests.

Received: 8 March 2011 Accepted: 20 October 2011

Published: 20 October 2011

\section{References}

1. Shin HR, Won YJ, Jung KW, Kong HJ, Yim SH, Lee JK, Noh HI, Pisani P, Park JG: Nationwide cancer incidence in Korea, 1999 2001; first result using the national cancer incidence database. Cancer Res Treat 2005, 37:325-331.

2. Esteva FJ, Hortobagyi GN: Locally advanced breast cancer. Hematol Oncol Clin North Am 1999, 13:457-472, vii.

3. Estevez LG, Gradishar WJ: Evidence-based use of neoadjuvant taxane in operable and inoperable breast cancer. Clin Cancer Res 2004, 10:3249-3261.

4. Kaufmann M, von Minckwitz G, Smith R, Valero V, Gianni L, Eiermann W, Howell A, Costa SD, Beuzeboc P, Untch M, Blohmer JU, Sinn HP, Sittek R, Souchon R, Tulusan AH, Volm T, Senn HJ: International expert panel on the use of primary (preoperative) systemic treatment of operable breast cancer: Review and recommendations. J Clin Oncol 2003, 21:2600-2608.

5. Couturier O, Jerusalem G, N'Guyen JM, Hustinx R: Sequential positron emission tomography using [18f]fluorodeoxyglucose for monitoring response to chemotherapy in metastatic breast cancer. Clin Cancer Res 2006, 12:6437-6443.

6. Hodgson NC, Gulenchyn KY: Is there a role for positron emission tomography in breast cancer staging? J Clin Oncol 2008, 26:712-720.

7. Lee JH, Rosen EL, Mankoff DA: The role of radiotracer imaging in the diagnosis and management of patients with breast cancer: Part 2response to therapy, other indications, and future directions. J Nucl Med 2009, 50:738-748.

8. Sloka JS, Hollett PD, Mathews M: A quantitative review of the use of fdgpet in the axillary staging of breast cancer. Med Sci Monit 2007, 13: RA37-46.

9. Emmering J, Krak NC, Van der Hoeven JJ, Spreeuwenberg MD, Twisk JW, Meijer S, Pinedo HM, Hoekstra OS: Preoperative [18f] fdg-pet after chemotherapy in locally advanced breast cancer: Prognostic value as compared with histopathology. Ann Oncol 2008, 19:1573-1577.

10. Kim SJ, Kim SK, Lee ES, Ro J, Kang S: Predictive value of [18f]fdg pet for pathological response of breast cancer to neo-adjuvant chemotherapy. Ann Oncol 2004, 15:1352-1357.

11. Krak NC, Hoekstra OS, Lammertsma AA: Measuring response to chemotherapy in locally advanced breast cancer: Methodological considerations. Eur J Nucl Med Mol Imaging 2004, 31(Suppl 1):S103-111.

12. Rousseau C, Devillers A, Sagan C, Ferrer L, Bridji B, Campion L, Ricaud M, Bourbouloux E, Doutriaux I, Clouet M, Berton-Rigaud D, Bouriel C, Delecroix V, Garin E, Rouquette S, Resche I, Kerbrat P, Chatal JF, Campone M: Monitoring of early response to neoadjuvant chemotherapy in stage ii and iii breast cancer by [18f] fluorodeoxyglucose positron emission tomography. J Clin Oncol 2006, 24:5366-5372.

13. Schelling M, Avril N, Nahrig J, Kuhn W, Romer W, Sattler D, Werner M, Dose J, Janicke F, Graeff H, Schwaiger M: Positron emission tomography using [(18)f]fluorodeoxyglucose for monitoring primary chemotherapy in breast cancer. J Clin Oncol 2000, 18:1689-1695.

14. Keam B, Im SA, Kim HJ, Oh DY, Kim JH, Lee SH, Chie EK, Han W, Kim DW, Cho N, Moon WK, Kim TY, Park IA, Noh DY, Heo DS, Ha SW, Bang YJ: Clinical significance of axillary nodal ratio in stage II/III breast cancer treated with neoadjuvant chemotherapy. Breast Cancer Res Treat 2009, 116:153-160.

15. Keam B, Im SA, Kim HJ, Oh DY, Kim JH, Lee SH, Chie EK, Han W, Kim DW, Moon WK, Kim TY, Park IA, Noh DY, Heo DS, Ha SW, Bang YJ: Prognostic 
impact of clinicopathologic parameters in stage ii/iii breast cancer treated with neoadjuvant docetaxel and doxorubicin chemotherapy: Paradoxical features of the triple negative breast cancer. BMC Cancer 2007, 7:203.

16. Bos R, van Der Hoeven JJ, van Der Wall E, van Der Groep P, van Diest PJ, Comans EF, Joshi U, Semenza GL, Hoekstra OS, Lammertsma AA, Molthoff CF: Biologic correlates of (18)fluorodeoxyglucose uptake in human breast cancer measured by positron emission tomography. J Clin Oncol 2002, 20:379-387.

17. Kong X, Moran MS, Zhang N, Haffty B, Yang Q: Meta-analysis confirms achieving pathological complete response after neoadjuvant chemotherapy predicts favourable prognosis for breast cancer patients. Eur J Cancer 2011, 47:2084-2090.

18. Kuerer HM, Newman LA, Smith TL, Ames FC, Hunt KK, Dhingra K, Theriault RL, Singh G, Binkley SM, Sneige N, Buchholz TA, Ross MI, McNeese MD, Buzdar AU, Hortobagyi GN, Singletary SE: Clinical course of breast cancer patients with complete pathologic primary tumor and axillary lymph node response to doxorubicin-based neoadjuvant chemotherapy. J Clin Oncol 1999, 17:460-469.

19. Therasse P, Arbuck SG, Eisenhauer EA, Wanders J, Kaplan RS, Rubinstein L, Verweij J, Van Glabbeke M, van Oosterom AT, Christian MC, Gwyther SG: New guidelines to evaluate the response to treatment in solid tumors. European organization for research and treatment of cancer, national cancer institute of the united states, national cancer institute of canada. J Natl Cancer Inst 2000, 92:205-216.

20. Recht A, Edge SB, Solin LJ, Robinson DS, Estabrook A, Fine RE, Fleming GF, Formenti S, Hudis C, Kirshner JJ, Krause DA, Kuske RR, Langer AS, Sledge GW Jr, Whelan TJ, Pfister DG: Postmastectomy radiotherapy: Clinical practice guidelines of the american society of clinical oncology. J Clin Oncol 2001, 19:1539-1569.

21. Han S, Kim SB, Kang SS, Noh WC, Paik NS, Chang ES, Kim JR, Lim SH, Park HS: A phase ii study of neoadjuvant docetaxel plus doxorubicin (kbcs-01) in stage ii, iii breast cancer. Breast Cancer Res Treat 2006, 98:57-61.

22. Lee KH, Im SA, Oh DY, Lee SH, Chie EK, Han W, Kim DW, Kim TY, Park IA, Noh DY, Heo DS, Ha SW, Bang YJ: Prognostic significance of bcl-2 expression in stage iii breast cancer patients who had received doxorubicin and cyclophosphamide followed by paclitaxel as adjuvant chemotherapy. BMC Cancer 2007, 7:63.

23. Perez EA, Roche PC, Jenkins RB, Reynolds CA, Halling KC, Ingle JN, Wold LE: Her2 testing in patients with breast cancer: Poor correlation between weak positivity by immunohistochemistry and gene amplification by fluorescence in situ hybridization. Mayo Clin Proc 2002, 77:148-154.

24. Carey LA, Dees EC, Sawyer L, Gatti L, Moore DT, Collichio F, Ollila DW, Sartor Cl, Graham ML, Perou CM: The triple negative paradox: Primary tumor chemosensitivity of breast cancer subtypes. Clin Cancer Res 2007, 13:2329-2334.

25. Honkoop AH, van Diest PJ, de Jong JS, Linn SC, Giaccone G, Hoekman K, Wagstaff J, Pinedo HM: Prognostic role of clinical, pathological and biological characteristics in patients with locally advanced breast cancer. Br J Cancer 1998, 77:621-626.

26. Mazouni C, Peintinger F, Wan-Kau S, Andre F, Gonzalez-Angulo AM, Symmans WF, Meric-Bernstam F, Valero V, Hortobagyi GN, Pusztai L: Residual ductal carcinoma in situ in patients with complete eradication of invasive breast cancer after neoadjuvant chemotherapy does not adversely affect patient outcome. J Clin Oncol 2007, 25:2650-2655.

27. Simon R: Optimal two-stage designs for phase II clinical trials. Control Clin Trials 1989, 10:1-10

28. Avril N, Rose CA, Schelling M, Dose J, Kuhn W, Bense S, Weber W, Ziegler S, Graeff $H$, Schwaiger M: Breast imaging with positron emission tomography and fluorine-18 fluorodeoxyglucose: Use and limitations. Clin Oncol 2000, 18:3495-3502.

29. Kumar A, Kumar R, Seenu V, Gupta SD, Chawla M, Malhotra A, Mehta SN: The role of (18)f-fdg pet/ct in evaluation of early response to neoadjuvant chemotherapy in patients with locally advanced breast cancer. Eur Radiol 2009.

30. Berriolo-Riedinger A, Touzery C, Riedinger JM, Toubeau M, Coudert B, Arnould L, Boichot C, Cochet A, Fumoleau P, Brunotte F: [18f]fdg-pet predicts complete pathological response of breast cancer to neoadjuvant chemotherapy. Eur I Nucl Med Mol Imaging 2007, 34:1915-1924.
31. Duch J, Fuster D, Munoz M, Fernandez PL, Paredes P, Fontanillas M, Guzman F, Rubi S, Lomena FJ, Pons F: (18)f-fdg pet/ct for early prediction of response to neoadjuvant chemotherapy in breast cancer. Eur I NuCl Med Mol Imaging 2009.

32. Dunnwald LK, Gralow JR, Ellis GK, Livingston RB, Linden HM, Specht JM, Doot RK, Lawton TJ, Barlow WE, Kurland BF, Schubert EK, Mankoff DA: Tumor metabolism and blood flow changes by positron emission tomography: Relation to survival in patients treated with neoadjuvant chemotherapy for locally advanced breast cancer. J Clin Oncol 2008 26:4449-4457.

33. Mankoff DA, Dunnwald LK, Gralow JR, Ellis GK, Schubert EK, Tseng J, Lawton TJ, Linden HM, Livingston RB: Changes in blood flow and metabolism in locally advanced breast cancer treated with neoadjuvant chemotherapy. J Nucl Med 2003, 44:1806-1814.

34. McDermott GM, Welch A, Staff RT, Gilbert FJ, Schweiger L, Semple SI, Smith TA, Hutcheon AW, Miller ID, Smith IC, Heys SD: Monitoring primary breast cancer throughout chemotherapy using fdg-pet. Breast Cancer Res Treat 2007, 102:75-84.

35. Schwarz-Dose J, Untch M, Tiling R, Sassen S, Mahner S, Kahlert S, Harbeck N, Lebeau A, Brenner W, Schwaiger M, Jaenicke F, Avril N: Monitoring primary systemic therapy of large and locally advanced breast cancer by using sequential positron emission tomography imaging with [18f]fluorodeoxyglucose. J Clin Oncol 2009, 27:535-541.

36. Smith IC, Welch AE, Hutcheon AW, Miller ID, Payne S, Chilcott F, Waikar S, Whitaker T, Ah-See AK, Eremin O, Heys SD, Gilbert FJ, Sharp PF: Positron emission tomography using [(18)f]-fluorodeoxy-d-glucose to predict the pathologic response of breast cancer to primary chemotherapy. J Clin Oncol 2000, 18:1676-1688.

37. Jung SY, Kim SK, Nam BH, Min SY, Lee SJ, Park C, Kwon Y, Kim EA, Ko KL, Park IH, Lee KS, Shin KH, Lee S, Kim SW, Kang HS, Ro J: Prognostic impact of [(18)f] fdg-pet in operable breast cancer treated with neoadjuvant chemotherapy. Ann Surg Oncol 2009.

38. Avril N, Menzel M, Dose J, Schelling M, Weber W, Janicke F, Nathrath W, Schwaiger M: Glucose metabolism of breast cancer assessed by $18 \mathrm{ff}-\mathrm{fdg}$ pet: Histologic and immunohistochemical tissue analysis. J Nucl Med 2001, 42:9-16.

39. Jung SY, Han W, Lee JW, Ko E, Kim E, Yu JH, Moon HG, Park IA, Oh DY, Im SA, Kim TY, Hwang KT, Kim SW, Noh DY: Ki-67 expression gives additional prognostic information on st. Gallen 2007 and adjuvant! Online risk categories in early breast cancer. Ann Surg Oncol 2009.

40. Basu S, Chen W, Tchou J, Mavi A, Cermik T, Czerniecki B, Schnall M, Alavi A: Comparison of triple-negative and estrogen receptor-positive/ progesterone receptor-positive/her2-negative breast carcinoma using quantitative fluorine-18 fluorodeoxyglucose/positron emission tomography imaging parameters: A potentially useful method for disease characterization. Cancer 2008, 112:995-1000

\section{Pre-publication history}

The pre-publication history for this paper can be accessed here: http.//www.biomedcentral.com/1471-2407/11/452/prepub

doi:10.1186/1471-2407-11-452

Cite this article as: Keam et al.: Early metabolic response using FDG

$\mathrm{PET} / \mathrm{CT}$ and molecular phenotypes of breast cancer treated with neoadjuvant chemotherapy. BMC Cancer 2011 11:452.

\section{Submit your next manuscript to BioMed Central and take full advantage of:}

- Convenient online submission

- Thorough peer review

- No space constraints or color figure charges

- Immediate publication on acceptance

- Inclusion in PubMed, CAS, Scopus and Google Scholar

- Research which is freely available for redistribution 